\title{
FRECUENCIA DE TRASTORNO DE LA CONDUCTA ALIMENTARIA EN ESTUDIANTES DE LA ESCUELA DE ARQUITECTURA DE LA UNIVERSIDAD CÉSAR VALLEJO, 2018
}

\author{
FREQUENCY OF DISORDER OF FOOD BEHAVIOR IN STUDENTS \\ OF THE SCHOOL OF ARCHITECTURE OF THE \\ UNIVERSITY CÉSAR VALLEJO, 2018
}

\author{
Vanessa Velásquez-Castillo ${ }^{1}$ Emilio Vega-Gonzales ${ }^{2}$, \\ Fiorella Cubas-Romero ${ }^{3}$
}

\section{RESUMEN}

Los trastornos de la conducta alimentaria (TCA) son patologías psicológicas caracterizadas por la crisis depresiva, estrés, ansiedad, el peso, y la insatisfacción corporal. Así mismo, son consideradas como principales problemas de salud mental en los jóvenes por su elevado crecimiento en esta población. El objetivo de la investigación fue determinar la frecuencia de trastornos de la conducta alimentaria en estudiantes de la Escuela de Arquitectura de la Universidad César Vallejo, para ello se realizó un estudio básico de nivel descriptivo, con un diseño no experimental-transversal, y se trabajó con una muestra de 86 estudiantes del I y II ciclo de la

1 Estudiante de la Escuela de Nutrición de la Universidad César Vallejo Lima Este. Dirección Postal: Av. del Parque 640, Urb. Canto Rey, San Juan de Lurigancho-Lima Perú. Correo electrónico: vcvane1218@gmail.com

2 Docente de la Escuela de Nutrición de la Universidad César Vallejo Lima Este. Dirección Postal: Av. del Parque 640, Urb. Canto Rey, San Juan de Lurigancho-Lima Perú. Correo electrónico: emilioswald@gmail.com

3 Coordinadora de la Escuela de Nutrición de la Universidad César Vallejo Lima Este. Dirección Postal: Av. del Parque 640, Urb. Canto Rey, San Juan de Lurigancho-Lima Perú. Correo electrónico: fcubas@ucv.edu.pe 
facultad, mediante la aplicación del cuestionario SCOFF(Sick, Control, One, Fat, Food). Los resultados evidencian una prevalencia de TCA del 21\% (SCOFF), con una frecuencia mayor en el sexo femenino (30,8\%) en comparación con el masculino (12,8\%).Se destaca además que el $84,9 \%$ refiere haber perdido recientemente más de $6 \mathrm{~kg}$ en un periodo de tres meses. Se concluye que hay una prevalencia moderada de TCA en la población universitaria, especialmente entre el sexo femenino.

PALABRAS CLAVE: Trastornos de la conducta alimentaria, SCOFF.

\section{ABSTRACT}

Eating disorders (eating disorders) are psychological pathologies characterized by depressive crisis, stress, anxiety, weight, and body dissatisfaction. Likewise, they are considered as the main mental health problems in young people due to their high growth in this population. The objective of the research was to determine the frequency of eating disorders in students of the School of Architecture at the César Vallejo University. For this purpose, a basic study was carried out at a descriptive level, with a non - experimental transversal design, and with a sample of 86 students of the I and II cycle of the faculty, by means of the application of the SCOFF questionnaire (Sick, Control, One, Fat, Food). The results show a prevalence of ACT of $21 \%$ (SCOFF), with a higher frequency in females $(30.8 \%)$ compared to males $(12.8 \%)$. It is also noted that $84.9 \%$ reported having recently lost more than $6 \mathrm{~kg}$ in a period of three months. It is concluded that there is a moderate prevalence of ED in the university population, especially among females.

KEY WORDS: Eating disorders, SCOFF.

\section{INTRODUCCIÓN}

Los TCA están vinculados a la preocupación por el peso y la insatisfacción corporal, generando importantes alteraciones conductuales. Dada su prevalencia, curso clínico prolongado y gravedad se consideran uno de los principales problemas de salud mental en los jóvenes. ${ }^{1}$ Con más del 90\% estos trastornos tienen mayor prevalencia entre las mujeres; sin embargo, la duración de los trastornos alimentarios es variable. En las estadísticas se observa también que el 30\% o 40\% que padece estos trastornos pone en riesgo su vida; y el 20\%, muere. ${ }^{2}$

El TCA más común es la anorexia, presente entre el 0.5\% al 3\% de adolescentes, cifras que pueden duplicarse si se consideran a adolescentes sanas con preocupación anormal por su peso corporal y conductas alimentarias anormales. Por otro lado, la bulimia se presenta en un 0.6 a un $13 \%$ de mujeres mayores de 18 años. $^{3}$ 
En el Perú, el Instituto Nacional de Salud Mental Honorio Delgado evidenció que la frecuencia de adolescentes padecen problemas de trastornos de conducta alimentaria representan entre el 7,3 y 11,4\% en una muestra de nueve mil adolescentes encuestados en 17 ciudades de nuestro país entre ellas, Lima, Tacna, Iquitos, Callao, Puno y Pucallpa. ${ }^{4}$

El Manual Diagnóstico y Estadístico de los Trastornos Mentales (DSM-5) afirma que entre los TCA se encuentran bulimia nerviosa (BN), anorexia nerviosa (AN), trastorno por atracón, pica, trastorno restrictivo de la ingesta y trastornos de la conducta alimentaria no especificados. ${ }^{5} \mathrm{La} \mathrm{BN}$ y AN presentan una tasa de morbilidad de $33 \%$, siendo las enfermedades infecciosas y arritmias sus principales causas de muerte.

La AN es considerada un trastorno caracterizado por la pérdida inducida, continua y deliberada de peso de una persona. Está asociada con una psicopatología específica que genera un temor ante la robustez y la flacidez de las formas corporales, quien padece este trastorno se impone a sí mismo un límite máximo de peso bajo, generando la mayoría de veces desnutrición, con cambios y alteraciones metabólicos y endocrinos secundarios de la función corporal. Entre algunos síntomas se encuentran una dieta restrictiva voluntaria y un excesivo ejercicio físico. ${ }^{7}$ En el caso de la bulimia nerviosa (BN), se observa una ingestión exagerada y repetitiva de alimentos, seguida de vómitos y uso de purgantes debido a una preocupación excesiva por el control del peso corporal, las cuales suelen producir daños a nivel oral y alteraciones electrolíticas. ${ }^{8}$

El trastorno por atracón (TPA) conocido desde el siglo XX, es un nuevo trastorno de comportamiento alimentario asociado frecuentemente con la obesidad y reconocido por las clasificaciones internacionales, aunque muy limitadas y relacionadas al componente compulsivo de la $\mathrm{BN}$, existen diversas fuentes de investigación que aportan información acerca de su perfil psicopatológico. Se caracteriza por el descontrol alimentario, en episodios donde sin realizar ninguna conducta compensatoria después, se ingiere una enorme cantidad de calorías y esta a su vez con una sensación de pérdida del control. ${ }^{9}$

Finalmente, en la categoría TCA no especificada se consideran a los TCA que no cumplen requisitos determinados para algún TCA específico. Por ejemplo: Algunas mujeres completan todos los criterios para diagnosticar la $\mathrm{BN}$, con la excepción de que los atracones aparecen menos del tiempo especificado (2 veces por semana o menos de 3 meses). Otras cumplen todos los criterios de la 
AN excepto que, a pesar de la significativa pérdida de peso, éste aún se encuentre dentro de los límites normales o las menstruaciones sean regulares. Incluso no tragar, sino masticar y expulsar cantidades considerables de comida. ${ }^{10}$

El incremento de las TCA en los últimos años, en especial entre la población adolescente y universitaria., está relacionado con la presencia de diversos factores, entre ellos el gran desconocimiento sobre una buena alimentación, el cual puede estar relacionado a su vez con la consulta de recursos online que no tienen validez científica alguna. ${ }^{11}$ Desde un punto de vista psicológico, las variaciones en la personalidad y los cuadros depresivos se han asociado con el desarrollo de trastornos por atracón en adolescentes con obesidad. ${ }^{12}$

Es importante resaltar que a pesar de realizarse relevantes investigaciones de pacientes con TCA, existen poblaciones en riesgo que experimentan y registran síntomas notables como realización de conductas nocivas para la salud e insatisfacción corporal y a pesar de eso no son diagnosticados a causa de la inflexibilidad y rigidez de criterios clínicos establecidos. Por lo tanto, se reconoce la urgente necesidad implementar y aplicar métodos de detección en los individuos más vulnerables, con el fin intervenir en prevención desde edades tempranas y adicionar educación de calidad referente a los TCA. ${ }^{13}$

Este trabajo de investigación se realiza debido al alto índice de adolescentes que padecen TCA, creando la necesidad de prevenir o disminuir su prevalencia incluyendo los efectos posteriores, e incentivar al personal de salud en la búsqueda de estrategias para reforzar la promoción y prevención de estos trastornos para permitir un mejor rendimiento y un óptimo estado de salud en los adolescentes. Por ello se plantea como objetivo determinar la frecuencia de trastorno de conducta alimentaria en estudiantes de la Escuela de Arquitectura de la Universidad César Vallejo, 2018.

\section{MÉTODOS}

\section{Diseño de investigación}

Se realizó una investigación básica de nivel descriptivo, con un diseño no experimental y corte transversal. La población del estudio estuvo formada por todos los alumnos de la Escuela Profesional de Arquitectura de la Universidad César Vallejo, con una muestra de 86 alumnos del Primer y Segundo ciclo de las secciones A y B de la Escuela Profesional de Arquitectura de la Universidad César Vallejo. La elección de la muestra se realizó un muestreo aleatorio simple. 


\section{Técnica e Instrumentos de recolección de datos}

En los TCA se requieren pruebas psicométricas que respaldan las observaciones de los profesionales que tienen contacto con los pacientes. Por lo tanto, se utiliza una serie de cuestionarios que evalúan distintos aspectos que influyen en este tipo de trastornos. Entre los cuestionarios más utilizados se encuentra el Sick Control On Fat Food (SCOFF) el cual aporta información relevante para determinar hábitos y pensamientos relacionados con la imagen corporal y la alimentación. ${ }^{14}$

El cuestionario SCOFF fue desarrollado por John Morgan y colaboradores en la Fundación "Leeds and York Partnership NHS Trust" y se caracteriza por tener cinco simples preguntas de selección las cuales han sido validadas en entornos de atención primaria y especializada. Con una sensibilidad de $100 \%$ y especificidad de $90 \%$ en cuanto a la AN, generando sospecha de un caso al considerar una puntuación de 2 o más respuestas positivas. ${ }^{15}$ Cada respuesta positiva equivale a un punto, una puntuación $>2$, se relaciona a un posible caso de TCA. Un estudio reciente demostró su validez cuando se aplica en estudiantes universitarios, obteniendo una sensibilidad de $78 \%$, una especificidad de $84 \%$, un valor predictivo positivo de $58 \%$ y un valor predictivo negativo de $93 \%$, usando como referencia el cuestionario EDI-2. ${ }^{16}$

\section{Aspectos éticos}

Los estudiantes universitarios fueron informados acerca de la investigación mediante un consentimiento informado, respetando en todo momento su anonimato, privacidad y el deseo voluntario de participar en el estudio.

\section{RESULTADOS}

El cuestionario SCOFF se realizó en cuatro salones de la Facultad de Arquitectura: I ciclo A, I ciclo B, II ciclo A, II ciclo B; donde se demostró que la población en riesgo abarcaba un 21\% y la población sin riesgo un 79\% del total de la muestra. (Gráfico 1)

$\mathrm{Al}$ analizar el cuestionario SCOFF se encontró que la presencia del ítem $\mathrm{N}^{\circ}$ 3 (iHa perdido recientemente más de $6 \mathrm{~kg}$ en un periodo de tres meses?), con un $84,9 \%$, es el que tiene mayor frecuencia entre los jóvenes que formaron parte del estudio. (Tabla 1) 
Según la prueba de Chi cuadrado (Tabla 3) se observó una asociación significativa entre la frecuencia de TCA y el sexo de los estudiantes $(p=0,041)$.

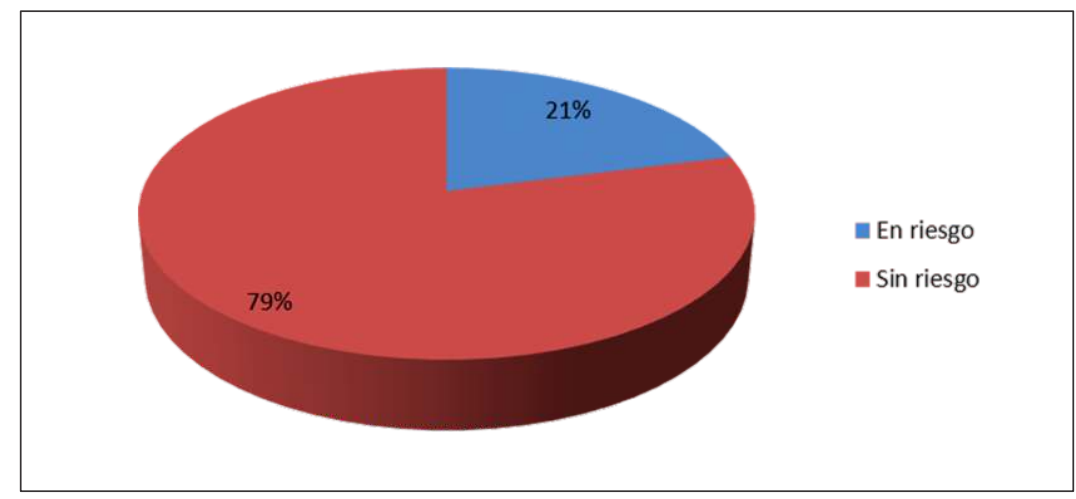

Gráfico 1. Prevalencia de TCA en estudiantes de Arquitectura de la Universidad César Vallejo según el cuestionario SCOFF.

Tabla 1

ITEMS DEL CUESTIONARIO SCOFF

\begin{tabular}{|c|c|c|c|c|c|}
\hline \multirow{2}{*}{\multicolumn{2}{|c|}{ Ítem }} & \multicolumn{2}{|c|}{$\mathrm{Si}$} & \multicolumn{2}{|c|}{ No } \\
\hline & & $\mathrm{n}$ & $\%$ & $\mathrm{n}$ & $\%$ \\
\hline \multirow[t]{2}{*}{1} & $\begin{array}{l}\text { ¿Tiene la sensación de estar enferma } \\
\text { porque siente el estómago tan lleno }\end{array}$ & & & & \\
\hline & que le resulta incómodo? & 70 & 81,4 & 16 & 18,6 \\
\hline \multirow[t]{2}{*}{2} & ¿Está preocupada/o porque siente que & & & & \\
\hline & tiene que controlarse cuando come? & 62 & 72,1 & 24 & 27,9 \\
\hline \multirow[t]{2}{*}{3} & ¿Ha perdido recientemente más de & & & & \\
\hline & $6 \mathrm{~kg}$ en un periodo de tres meses? & 73 & 84,9 & 13 & 15,1 \\
\hline \multirow[t]{2}{*}{4} & ¿Cree que está gorda/o aunque otros & & & & \\
\hline & digan que está demasiado delgada? & 71 & 82,6 & 15 & 17,4 \\
\hline 5 & ¿Diría que la comida domina su vida? & 71 & 82,6 & 15 & 17,4 \\
\hline
\end{tabular}


Tabla 2

RELACIÓN ENTRE EL SEXO DE LOS ESTUDIANTES DE ARQUITECTURA Y LAPRESENCIA DE TCA

\begin{tabular}{|c|c|c|c|c|c|c|c|}
\hline \multirow{3}{*}{ Ítem } & & \multicolumn{4}{|c|}{ Sexo } & \multirow{3}{*}{$x^{2}$} & \multirow{3}{*}{$p$ (sig.) } \\
\hline & & \multicolumn{2}{|c|}{$\mathrm{F}$} & \multicolumn{2}{|c|}{$M$} & & \\
\hline & & $\mathrm{n}$ & $\%$ & $n$ & $\%$ & & \\
\hline \multirow[t]{2}{*}{ SCOFF } & Sin riesgo & 27 & 69,2 & 41 & 87,2 & & \\
\hline & Con riesgo & 12 & 30,8 & 6 & 12,8 & 4,174 & 0,041 \\
\hline Total & & 39 & 100,0 & 47 & 100,0 & & \\
\hline
\end{tabular}

\section{DISCUSIÓN}

Considerando la puntuación obtenida con el cuestionario SCOFF, se observa en los estudiantes de Arquitectura una frecuencia de 21\% con riesgo de TCA, resultado ligeramente inferior al 30,1\% reportado por Fajardo et al. ${ }^{19}$ en adolescentes de Colombia. Al compararse este resultado con los reportados con el empleo de otro instrumento, como el EAT-26, se puede apreciar que este último muestra menores porcentajes, como el $15,6 \%$ reportado por Vega y col. ${ }^{20}$ y el $10,1 \%$ encontrado por Ponce et al..$^{21}$, en las escuelas deNutrición y Medicina de distintas universidades peruanas, respectivamente. Esto demuestra que el cuestionario SCOFF, el cual solo consta de cinco ítems, suele detectar un mayor número de estudiantes con riesgo, en comparación con el EAT-26; aunque debe tomarse en cuenta también la carrera a la que pertenecen los estudiantes. Los autores mencionados coinciden en la importancia que tiene la insatisfacción con la imagen corporal para el desarrollo de estos trastornos, tal como se observa en el ítem 4 del cuestionario SCOFF en el que un $82,7 \%$ de los estudiantes encuestados cree que está gorda/o aún cuando sus compañeros dicen que está demasiado delgada.

Otros autores como Saucedo et al. $^{22}$ afirman que la insatisfacción con la imagen corporal; no es un factor determinante para desarrollar TCA en la adolescencia, puesto que encontró en su estudio un $85 \%$ de estudiantes con insatisfacción corporal y apenas el 3,2\% presentaronconductas alimentarias de riesgo. Es posible que durante la etapa universitaria, un grupo mayoritario de alumnos haya priorizado más la parte académicay la necesidad de incrementar sus conocimientos para mejorar su imagen, que el hecho de mejorar su aspecto físico, desplazan- 
do así la búsqueda de los modelos estéticos dominantes que determina en los adolescentes en general la insatisfacción corporal.

Analizando el ítem 3 del cuestionario SCOFF, se aprecia un elevado porcentaje de estudiantes que reporta haber perdido recientemente más de $6 \mathrm{~kg}$ en un periodo de tres meses con un $84,9 \%$ así como un $82,6 \%$ que se considera gorda. La existencia de ambos resultados podría indicar la presencia de comportamientos restrictivos en la alimentación, los cuales podrían ser manifestaciones iniciales de cuadros anoréxicos y bulímicos, o predisponer a los estudiantes a autolesionarse en un futuro cercano. Este hallazgo podría reforzar las conclusiones a las que llegaron Martínez et al. ${ }^{23}$, quienes reportaron una asociación significativa, aunque débil, entre la presencia de trastornos alimentarios y el desarrollo de factores de riesgo para la ideación suicida. El factor familiar también puede desempeñar un rol importante en estos comportamientos restrictivos, y es posible que esta exigencia sea mayor entre los adolescentes cuyas familias tengan mayor poder adquisitivo y nivel educativo, como reportaron $\mathrm{Yu}$ et al. ${ }^{24}$. Es posible que los padres con estas características sean más exigentes con sus hijos en lo concerniente a su imagen corporal, y presionen a sus hijos para practicar dietas, no necesariamente adecuadas para su edad, y sin el apoyo psicológico de respaldo para no afectar su autoestima. Es posible que una forma de eludir esta presión se visibilice en la "sensación de estar enferma porque siente el estómago tan lleno que le resulta incómodo", correspondiente al ítem 1del cuestionario SCOFF, el cual, en el presente estudio, fue contestado de manera afirmativa por el $81,4 \%$ de los estudiantes encuestados.

El ítem $\mathrm{N}^{\circ} 2$, que indica si el estudiante está preocupada/o porque siente que tiene que controlar cuando come, presentó una frecuencia de respuestas positivas del $72,1 \%$, resultado que coincide con las conclusiones reportadas por Beníte $z^{25}$ quien afirmó que la mayor prevalencia de TCA va relacionada con la imagen y el Índice de Masa Corporal (IMC). Se entiende por tanto que el tener un bajo peso o la percepción de un cuerpo delgado suele estar involucrado con el riesgo de TCA y la búsqueda de bajar de peso en poco tiempo. La relación también ha sido reportada por Fernández $z^{3}$,quien determinó la existencia de una relación entre el control oral y la percepción de la imagen corporal.

En la tabla 3 se observa que el sexo femenino tiene mayor prevalencia de riesgo de TCA en proporción aproximada de 2 a 1. Los resultados más comunes en la literatura científica coinciden con este hallazgo, como en el estudio de Álva- 
rez, Bautista y Serra (26) realizado en adolescentes españoles, con una prevalencia global de TCA de $4,11 \%$ distribuido en $5,46 \%$ de mujeres y $2,55 \%$ de hombres, así como el obtenido por Keski y Musteli ${ }^{27}$ donde las mujeres tenían una prevalencia de TCA de 2-3\% a diferencia de los hombres que solo llegaban al 0,3 $-0,7 \%$. En gran medida esta diferencia se aprecia más entre adolescentes y jóvenes, debido a la preocupación que ellos tienen por su apariencia física, la cual representa en gran medida la imagen que tendrán para la sociedad y especialmente para sus pares.

Para explicar este fenómeno de la diferencia de resultados entre hombres y mujeres en torno a las TCA, se debe mencionar lo reportado por Granero et al. ${ }^{28}$, quién llegó a la conclusión de que los varones están más preocupados por la masa muscular que tiene su cuerpo, a diferencia de las mujeres quienes suelen buscar una apariencia más delgada y esbelta. Otro estudio que se suma a esta insatisfacción de la imagen corporal, aunque con un resultado opuesto, ya que menciona una mayor frecuencia de insatisfacción en el sexo masculino, es el de Cruz et al. ${ }^{29}$. Los referidos autores encontraron un $20.8 \%$ de insatisfacción en hombres en comparación con el $15.9 \%$ de las mujeres, rompiendo el esquema tradicional de que la problemática de la imagen corporal así como los trastornos alimentarios que de este deriva son exclusivamente femeninas.

Sin embargo, en las últimas décadas los estudios en población masculina han mostrado un gradual aumento en la preocupación por la imagen corporal, producto, entre otros factores, de la influencia que ejercen en los adolescentes el incremento de la difusión de imágenes masculinas estereotipadas en los medios de comunicación. Un estudio que apoya esta idea, es el de Hernández y Londaño ${ }^{30}$ aunque no obtiene un resultado contundente como para afirmar que el sexo masculino está asociado a una mayor frecuencia de TCA, lo cual podría explicarse por una valoración distinta a la imagen corporal en comparación con la importancia que le dan a su alimentación. Mientras las mujeres, aparentemente, colocan a su imagen corporal por encima de su alimentación, ya que para obtener un cuerpo esbelto deben reducir la cantidad de alimentos que ingieren, en los varones, la alimentación es clave para la obtención de una mayor masa muscular, siendo más probable de que adquieran sobrepeso en vez de cuadros de anorexia y bulimia.

Un estudio en Colombia, realizado por Becerra et al. ${ }^{31}$ aporta otra explicación a esta diferencia entre los sexos masculino y femenino. De acuerdo a sus resultados, la tendencia del sexo femenino de predominio en la frecuencia de 
TCA se da a medida que avanza la etapa escolar, así, en los grados inferiores es más común ver estos problemas entre los varones. Por ello la identificación de las TCA no debe limitarse a la adolescencia tardía, sino que debe ser abordada desde las etapas tempranas a través de estrategias preventivas multidisciplinarias que refuercen los distintos aspectos de la personalidad aún inmadura del adolescente.

\section{CONCLUSIÓN}

La frecuencia de TCA en los estudiantes de Arquitectura en la Universidad César Vallejo es del 21\% según el cuestionario SCOFF, aunque este resultado puede modificarse de acuerdo al instrumento utilizado, por lo que se recomienda realizar estudios comparativos en futuras investigaciones. La mayor frecuencia fue detectada entre las estudiantes del sexo femenino, en una proporción de dos a uno en comparación con los del sexo masculino. Se recomienda la implementación de estrategias para detectar estos trastornos, con el fin de poder intervenir oportunamente y evitar el desarrollo de estadios más complicados, asimismo trabajar en la prevención en las poblaciones más vulnerables.

\section{REFERENCIAS BIBLIOGRÁFICAS}

1. Escolar MC, Martínez MÁ, González MY, Medina MB, Mercado E, Lara F. Risk factors of eating disorders in university students: Estimation of vulnerability by sex and age. Revista Mexicana de Trastornos Alimentarios (Internet) 2017 (Citado el 28 marzo 2019);8(2):10512. Disponible en:http://www.scielo.org.mx/pdf/rmta/v8n2/2007-1523-rmta-8-0200105.pdf

2. Micali N, Holliday J, Karwautz A, Haidvogl M, Wagner G, Fernandez-Aranda F, et al. Childhood eating and weight in eating disorders: a multi-centre European study of affected women and their unaffected sisters. Psychotherapy and psychosomatics (Internet) 2007 (Citado el 28 marzo 2019); 76(4):234-41. Disponible en:

https://www.karger.com/Article/Abstract/101502

3. Fernández C. Relación entre la percepción de la imagen corporal y los signos y síntomas de los trastornos del comportamiento alimentario en las alumnas del 3er al 5 to año de la Institución Educativa Nacional. Ate - Vitarte, 2016 Lima: Universidad Peruana Unión; 2017. (Citado el 28 marzo 2019) Disponible en: http://repositorio.upeu.edu.pe/handle/UPEU/675

4. Instituto Nacional de Salud Mental Honorio Delgado-Hideyo Noguchi. Trastornos alimenticios en adolescentes del Perú. Lima: Ministerio de Salud; 2010.

5. American Psychiatric Association. Diagnostic and Statistical Manual of Mental Disorders. DSM-5 (5th edition). Washington DC.: Editorial Médica Panamericana; 2013. 
6. Rivera MT, Parra MdS, Barriguete JA. Trastornos de la conducta alimentaria como factor de riesgo para osteoporosis. Salud publica de Mexico (Internet) 2005 (Citado el 28 marzo 2019);47(4):308-18. Disponible en: http://www.scielo.org.mx/scielo.php?script $=$ sci arttext\&pid $=$ S0036-36342005000400009

7. Cuadro E, Baile JI. Binge eating disorder: analysis and treatment. Revista Mexicana de Trastornos Alimentarios - Mexican Journal of Eating Disorders (Internet) 2015 (Citado el 28 marzo 2019);6(2):97-107. Disponible en:

http://journals.iztacala.unam.mx/index.php/amta/article/view/345

8. Rosten A, Newton T. [The impact of bulimia nervosa on oral health: A systematic review of the literature]. Nederlands tijdschrift voor tandheelkunde (Internet) 2019(Citado el 28 marzo 2019);126(3):141-50.. De invloed van boulimia nervosa op de mondgezondheid: een systematisch literatuuronderzoek. Disponible en:

https://www.nature.com/articles/sj.bdj.2017.837

9. Bolaños P. Cuestionarios usados en pacientes con trastornos de la conducta alimentaria. Trastornos de la Conducta Alimentaria (Internet) 2017 (Citado el 28 marzo 2019) 26:281021. Disponible en:https://dialnet.unirioja.es/servlet/articulo?codigo $=6433396$

10. Magariños M, García P. Guía de bolsillo de la Clasificación CIE-10. España: Editorial Médica Panamericana; 2000.

11. Behar R. La construcción cultural del cuerpo: El paradigma de los trastornos de la conducta alimentaria. Revista chilena de neuro-psiquiatría (Internet) 2010 (Citado el 28 marzo 2019);48(4):319-34. Disponible en:

https://scielo.conicyt.cl/scielo.php? script=sci_arttext\&pid=S0717-92272010000500007

12. Carriere C, Michel G, Feart C, Pellay H, Onorato O, Barat P, et al. Relationships between emotional disorders, personality dimensions, and binge eating disorder in French obese adolescents. Archives de pediatrie: organe officiel de la Societe francaise de pediatrie (Internet) 2019. (Citado el 28 marzo 2019) Disponible en:

https://www.sciencedirect.com/science/article/pii/S0929693X19300326?via\%3Dihub

13. Berengüí R, Castejón MÁ, Torregrosa MS. Body dissatisfaction, risk behaviors and eating disorders in university students. Revista Mexicana de Trastornos Alimentarios (Internet) 2016 (Citado el 28 marzo 2019); 7 (1):1-8. Disponible en:

https://www.sciencedirect.com/science/article/pii/S2007152316300052

14. Morgan JF, Reid F, Lacey JH. The SCOFF questionnaire: assessment of a new screening tool for eating disorders. BMJ (Internet) 1999 (Citado el 28 marzo 2019);319(7223):1467-8. Disponible en: https://www.bmj.com/content/319/7223/1467.long

15. Perry L, Morgan J, Reid F, Brunton J, O'Brien A, Luck A, et al. Screening for symptoms of eating disorders: reliability of the SCOFF screening tool with written compared to oral delivery. The International journal of eating disorders (Internet) 2002 (Citado el 28 marzo 2019);32(4):466-72.Disponible en:

https://onlinelibrary.wiley.com/doi/abs/10.1002/eat.10093

16. Sanchez O, Raffaelli M, Andrade FCD, Wiley AR, Noyola ANM, Arguelles AC, et al. Validation of the SCOFF questionnaire for screening of eating disorders among Mexican university 
students. Eating and weight disorders : EWD (Internet) 2017(Citado el 28 marzo 2019); 22(1):153-60. Disponible en:https://ink.springer.com/article/10.1007\%2Fs40519-016-0259-7

17. Garner DM, Garfinkel PE. The Eating Attitudes Test: an index of the symptoms of anorexia nervosa. Psychological medicine (Internet) 1979 (Citado el 28 marzo 2019);9(2):273-9. Disponible en: https://www.cambridge.org/core/journals/psychologicalmedicine/article/eating-attitudes-test-an-index-of-the-symptoms-of-anorexianervosa/084933330F96DB483B65BFE31E84856E

18. Gandarillas A, Zorrilla B, Sepúlveda A. Trastornos del comportamiento alimentario: Prevalencia de casos clínicos en mujeres adolescentes de la Comunidad de Madrid. Madrid: Documentos Técnicos de Salud Pública 2003.

19. Fajardo E, Méndez C, Jáuregui A. Prevalencia del riesgo de trastornos de la conducta alimentaria en una población de estudiantes de secundaria, Bogotá-Colombia. Rev.Fac.Med (Internet) 2017; 25 (1): 46-57. Disponible en: http://www.scielo.org.co/scielo.php? script $=$ sci_arttext\&pid $=$ S0121-52562017000100005\&lng $=$ en.

20. Vega E, Mosquera Z, Cubas F. Frecuencia de trastornos alimentarios en estudiantes de la escuela de nutrición de la Universidad César Vallejo Lima este, 2017. Revista UCV-Scientia Biomédica/Journalof Medicine and BiomedicalSciences (Internet) 2018;1(2):69-73. Disponible en: http://181.224.246.204/index.php/UCVSCIENTIABIOMEDICA/article/view/1822

21. Ponce C, Turpo K, Salazar C, Viteri L, CarhuanchoJ, Taype A. Trastornos de la conducta alimentaria en estudiantes de medicina de una universidad de Perú. Rev Cubana Salud Pública (Internet) 2017; 43(4). Disponible en: http://scielo.sld.cu/scielo.php? script $=$ sci_arttext\&pid $=$ S0864-34662017000400006\&lng $=$ es.

22. Saucedo T, J. Z, Villalón L, Peña A, León R. Prevalencia de factores de riesgo asociados a trastornos alimentarios en estudiantes universitarios hidalguenses. Psicología y Salud (Internet) 2015 (Citado el 28 marzo 2019);25(2):243-51. Disponible en:

https://biblat.unam.mx/es/revista/psicologia-y-salud/articulo/prevalencia-de-factores-deriesgo-asociados-a-trastornos-alimentarios-en-estudiantes-universitarios-hidalguenses

23. Martínez L, Vianchá MA, Pérez MP\& Avendaño BL. Asociación entre conducta suicida y síntomas de anorexia y bulimia nerviosa en escolares de Boyacá, Colombia. (Internet) Acta Colombiana de Psicología. 2017; 20 (2): 189-199. Disponible en:

http://www.scielo.org.co/scielo.php?pid $=$ S0123-91552017000200189\&script $=$ sci abstract\&tlng $=$ es

24. Yu J, Lu M, Tian L, Lu W, Meng F, Chen C. et al. Prevalence of disordered eating attitudes among University students in Wuhu, China. Nutr. Hosp. (Internet) 2015; 32 (4): 1752-1757. Disponible en: http://scielo.isciii.es/scielo.php?script $=$ sci_arttext\&pid $=$ S0212$16112015001000048 \& \operatorname{lng}=$ es.

25. Benítez A. Hábitos alimentarios de riesgo en la población universitaria extremeña: estudio efectuado en estudiantes de la Universidad de Extremadura. . España: Universidad de Extremadura, Departamento de Psicología y Antropología; 2016 Disponible en: http://dehesa.unex.es/bitstream/handle/10662/4087/TDUEX_2016_Benitez_Benitez.pdf? sequence $=1$ \&isAllowed $=\mathrm{y}$ 
26. Álvarez ML, Bautista I, Serra L. Prevalencia de los trastornos de la conducta alimentaria en adolescentes de Gran Canaria. Nutricion hospitalaria (Internet) 2015;31(5):2283-8.

Disponible en: http://scielo.isciii.es/scielo.php?script $=$ sci_arttext $\&$ pid $=$ S0212. 16112015000500050

27. Keski A, Mustelin L. Epidemiology of eating disorders in Europe: prevalence, incidence, comorbidity, course, consequences, and risk factors. Curr Opin Psychiatry (Internet) 2016; 29 (6):340-5. Disponible en: https://insights.ovid.com/pubmed?pmid $=27662598$

28. Granero A, Albo JM, Sicilia A, Medina J, Alcaraz M. Análisis de los estereotipos socioculturales hacia cuerpo delgado y cuerpo musculoso: diferencias en función del sexo y discrepancia con el peso. Revista de Psicodidáctica (Internet) 2018; 23 (1): 26-32. Disponible en: https://www.sciencedirect.com/science/article/pii/S1136103417301028

29. Cruz RM, Ávila ML, Velázquez HJ, Estrella DF. Evaluación de factores de riesgo de TCA en estudiantes de nutrición. Rev. Mex. de trastor. aliment (Internet) 2013; 4(1):37-44. Disponible en: http://www.scielo.org.mx/scielo.php?script $=$ sci_arttext $\&$ pid $=$ S2007 . $15232013000100005 \& \operatorname{lng}=$ es.

30. Hernandez L, Londaño C. Imagen corporal, IMC, afrontamiento, depresión y riesgo de TCA en jóvenes universitarios. Anales de psicología (Internet) 2013; 29 (3): 748-761 Disponible en: http://scielo.isciii.es/pdf/ap/v29n3/psicologia_clinica4.pdf

31. Becerra F, Saavedra Y, Verano A, \&Wilchez P. Riesgo de trastornos del comportamiento alimentario en un grupo de estudiantes de secundaria en un colegio de Bogotá D.C., Colombia. 2016. Revista de la Facultad de Medicina (Internet)2018. 66 (4), 571-574. Disponible en: https://revistas.unal.edu.co/index.php/revfacmed/article/view/62717/69155 